
cultures

Les cahiers de l'Acedle

10-2 | 2013

Apprendre les langues autrement

\title{
Testing et certification
}

\section{Claire Tardieu}

\section{OpenEdition \\ Journals}

Édition électronique

URL : http://journals.openedition.org/rdlc/1655

DOI : $10.4000 /$ rdlc. 1655

ISSN : 1958-5772

Éditeur

ACEDLE

\section{Référence électronique}

Claire Tardieu, «Testing et certification », Recherches en didactique des langues et des cultures [En ligne], 10-2 | 2013, mis en ligne le 07 juin 2013, consulté le 02 mai 2019. URL : http://

journals.openedition.org/rdlc/1655; DOI : 10.4000/rdlc.1655

Ce document a été généré automatiquement le 2 mai 2019

\section{(c) (i) $\odot$}

Recherches en didactique des langues et des cultures is licensed under a Creative Commons AttributionNonCommercial-NoDerivatives 4.0 International License 


\title{
Testing et certification
}

\author{
Claire Tardieu
}

\section{Introduction}

$1 \mathrm{Au}$ sein de la thématique "Apprendre les langues autrement", cet article se focalise sur la question de la certification en langues au moment où les modes d'évaluation ont tendance à évoluer et où des certifications européennes et internationales viennent supplanter les diplômes nationaux. On s'intéressera en particulier à deux certificats que tout semble opposer : le Certificat des Langues de l'Enseignement Supérieur (Cles) et le C-Test (forme spécifique de l'exercice de closure), qui incarnent chacun à leur façon une manière de certifier "autrement".

2 On se demandera d'abord dans quelle mesure le Cles qui emprunte ses fondements théoriques à l'approche actionnelle et à l'apprentissage par la tâche (Ellis, 2003; Bachman, 2007) propose réellement un mode de certification "autre" par rapport à l'approche structuro-psychométrique. Sur quelle conception de la compétence langagière repose-t-il véritablement? Sa difficulté à s'imposer en France peut-elle s'expliquer par d'autres raisons que des raisons politiques ou économiques? La rupture avec les autres modes est-elle aussi radicale qu'il y paraît ? Son approche est-elle réellement holistique ?

Paradoxalement, le C-Test (Grotjahn, 2006), peu utilisé et semblant s'inscrire à rebours des évolutions contemporaines favorables à une approche sociolinguistique intégrative (incluant l'expression et l'interaction orale), ne fait-il pas appel à l'hypothèse d'une compétence linguistique unitaire (Oller, 1976; Canale et Swain, 1980; Bachman, 1990), corroborée par le principe de l'unité de la cognition (Anderson, 1996)?

4 Si l'on comprend que l'on veuille donner à la certification une forme qui justifie un apprentissage des langues plus complet en amont, pour autant cette forme a minima du testing qu'est le C-test est-elle à rejeter totalement? Comme le montre la thèse récente de Reichert (2011), d'une certaine manière, aussi étrangers l'un à l'autre que soient le Cles et le C-Test, ils pourraient s'avérer compatibles, voire complémentaires.

5 Après un rappel de l'histoire du testing, on s'intéressera aux certificats de quatrième génération tels que le Cles et à leur apport, enfin on situera le C-Test par rapport au Cles. 


\section{L'histoire du testing}

6 Spolsky (1981) divise l'histoire du testing en langues en trois grandes approches: préscientifique, structuraliste psychométrique et sociolinguistique-intégrative (cf. Reichert, $2011: 38-39)$.

\section{L'approche pré-scientifique}

7 L'approche pré-scientifique s'inscrit dans une logique d'examens et de juges, mais manque d'objectivité et de fiabilité - la fiabilité ou fidélité étant l'assurance que l'on obtiendra des résultats similaires au même test si on le répète. (Par résultats similaires, il faut entendre le même classement des candidats et non le même résultat par candidat). Cette approche manque aussi de validité - une activité évaluative est valide si on a "la garantie qu'elle mesure exclusivement et exactement ce qu'elle est censée mesurer" (Horner, 2010: 17). La validité s'appuie sur la notion de construct, soit ce qui selon le contexte est visé par l'évaluation (la compétence de production écrite ou de compréhension de l'oral, par exemple). L'approche pré-scientifique trouve une illustration dans l'examen du Baccalauréat qui ne s'appuie ni sur des théories du langage ou de l'apprentissage, ni sur une méthodologie scientifique. Dès la première moitié du vingtième siècle, la docimologie (en particulier la commission Carnegie de 1932) a montré qu'il faudrait 127 correcteurs pour obtenir la valeur stable d'une dissertation de philosophie, 78, pour une composition française, 28 pour un devoir d'anglais, 19 pour une version latine et 13 pour un devoir de mathématiques... (Laugier \& Piéron, 1938; Piéron, $1963: 23)$.

\section{L'approche structuraliste-psychométrique}

L'approche structuraliste-psychométrique intervient pour répondre à la demande d'objectivité et de fiabilité, dans un souci d'évaluer la maitrise du système linguistique (Mc Namara, 2000), avec en contrepartie un effet d'atomisation et de décontextualisation. On trouve trace de cette approche dans une partie de l'épreuve du baccalauréat appelée "épreuve de compétence linguistique" instaurée en 1984 (B.O.E.N. n 27 du 7 juin 1983) et abolie en 2002 (B.O.E.N. $n^{\circ} 23$ du 7 juin 2001) soit des questions de nature grammaticale déconnectées du support.

\section{L'approche sociolinguistique intégrative}

9 A partir des années 60 , la demande est en faveur d'une approche intégrée, testant à la fois la compétence linguistique fine et les savoir-faire ( $\mathrm{CO}, \mathrm{CE}, \mathrm{PO}, \mathrm{PE})$ malgré les difficultés en termes de coût et d'attribution de scores en productions orales ou écrites.

Le Toefl est un bon exemple de test qui associe l'approche structuro-psychométrique avec des items discrets et l'approche intégrée introduisant les skills (savoir-faire de réception et de production). examinateurs ne disposent que d'un temps limité, ne voient qu'un échantillon limité de 
performances et qu'il y a des limites au nombre et à la nature des catégories qu'ils peuvent manipuler comme critères" (Horner, $2010: 22$ ).

Alors pourquoi une quatrième approche? Quels besoins nouveaux se sont-ils fait jour, besoins auxquels le Cles prétend répondre?

\section{Les limites de l'approche sociolinguistique intégrative}

13 La critique de Bachman (1990 : 82) à l'égard de ces tests alliant savoir-faire et éléments discrets (séparés) porte sur le fait qu'il est impossible de déterminer la nature du lien entre les deux. Ensuite, ce type d'évaluation ne mesure que certains aspects de la compétence à communiquer langagièrement. On peut citer à cet égard la synthèse réalisée par Springer (2000) dans sa "marguerite de la communication" et bien sûr les travaux d'oller (1976) et Bachman (1990). Après avoir passé en revue un certain nombre de travaux, Springer aboutit à une figure où la composante stratégique est au centre, les autres composantes (linguistique, socioculturelle, sociolinguistique, conversationnelle et discursive) se répartissant tout autour. Il organise dans ce modèle les apports de Hymes (composante socio-culturelle), de Charaudeau qui définissait une compétence "sémiolinguistique" à la fois d'ordre linguistique, situationnelle et discursive, de KerbratOrecchioni dont l'approche "pose la compétence conversationnelle comme "archicompétence" (Springer, 2000: 69), enfin, de Canale et Swain qui y ajoutent une composante stratégique.

Oller, quant à lui, affirme que la compétence langagière n'est pas seulement constituée d'éléments séparés, d'où l'idée d'une compétence intégrative : "the Unitary Competence Hypothesis." Même si ce concept est différent de celui de compétence de communication évoqué ci-dessus, pour oller, la production écrite ou orale n'est pas séparée de la réception, car le locuteur doit en permanence contrôler sa production pour s'assurer d'être compris. L'inverse étant tout aussi vrai : en phase de réception d'un message, l'auditeur ou le lecteur doivent formuler des hypothèses sur la suite du message. Par leur forme discrète, les tests formalisés ne mesurent pas cette dimension unitaire définie par Oller. Et ils ne mesurent pas davantage les dimensions sociologiques et pragmatiques de la communication, ni les dimensions cognitive et intellectuelle et encore moins la capacité de communication réelle chère à Bachman. Bachman fait en effet la distinction entre la compétence à communiquer langagièrement (communicative language ability) et la communication véritable (actual communication). La première inclut une dimension liée aux savoirs "language knowledge dimension" comprenant elle-même des compétences langagières organisationnelles et pragmatiques, des compétences stratégiques et des mécanismes psycho-physiologiques; la seconde se caractérise par sa dimension "d'utilisation réelle" (Bachman, 2007: 54). Bolton constatait déjà en 1991, avec l'avènement de l'approche communicative dans l'enseignement-apprentissage des langues, qu'il est beaucoup plus facile d'évaluer des connaissances que des compétences, $a$ fortiori lorsque ces dernières s'exercent dans un travail collaboratif et, conviendrait-il d'ajouter, "proche de la vie réelle."

15 Pour compléter la classification de Spolsky, une quatrième approche, non psychométrique, devrait être ajoutée : "l'approche sociolinguistique intégrative de type actionnel." Cette approche s'appuie sur une conception socio-pragmatique de la communication appliquée à l'enseignement dont on trouve les fondements chez 
Widdowson, Nunan et Ellis, entre autres. On pourrait inclure le Certificat de Compétences en Langues de l'Enseignement Supérieur (désormais Cles) dans cette catégorie.

\section{L'approche sociolinguistique intégrative de type actionnel}

\section{Les fondements}

Avec l'article 3 du décret $n^{\circ}$ 2005-1011 du 22 août 2005, l'institution commence à envisager un type de certification en langues qui ne soit pas directement connectée à l'apprentissage. Pour reprendre la taxonomie de Alderson (2002), il s'agit ici de faire la distinction entre le test de type "achievement" relié à un apprentissage en amont et le test de type "proficiency", indépendant d'une formation et tourné vers l'avenir. Ainsi fera-t-on mieux la distinction entre l'évaluation du savoir (ou du niveau) qui est "l'évaluation de l'atteinte d'objectifs spécifiques", "centrée sur le cours", correspondant à "une vue de l'intérieur" et l'évaluation de la capacité (mise en œuvre de la compétence ou performance), c'est-à-dire "de ce que l'on peut faire ou de que l'on sait en rapport avec son application au monde réel" (Cecrl, 2001: 139), l'évaluation de la capacité correspondant "à une vue de l'extérieur". Certains certificats existent déjà, comme le Deutsches Sprachdiplom (certificat d'allemand) proposé aux élèves volontaires en fin de troisième, ou le Certificat en Langue de l'Enseignement Supérieur (Cles) plus ou moins obligatoire à l'issue du master.

\section{Le CLES}

\section{Définition}

Inauguré en 2000 (voir B. $0 n^{\circ} 25$ du 29 juin 2000), révisé en 2007 (voir B.O. n 20 du 17 mai 2007), et inscrit, avec le C2i2e, comme un passage plus ou moins obligé des concours d'enseignement (malgré le décret $n^{\circ} 2012-999$ du 27 août 2012 abrogeant le décret $n$ - 2012-702 du 7 mai 2012), en quoi consiste exactement le Certificat de Compétences en Langues de l'Enseignement Supérieur?

Le CLES se décline en trois niveaux de qualification (1er, 2ème, 3ème degrés) définis respectivement par référence aux niveaux $B 1, B 2$ et $C 1$ du Conseil de l'Europe. Le 1er degrése situe au-delà du niveau d'exigence moyen de la langue vivante 1 au baccalauréat (B.O. $n^{\circ} 25$ du 29 juin 2000 : annexe 1 ).

Il est important de souligner ici que le niveau B2 du Cecrl, officiellement visé en fin de terminale, est également celui visé par le Cles 2 et non le Cles 1 . Ce décalage opéré dans le texte officiel peut s'expliquer de deux manières: tout d'abord, "le niveau d'exigence moyen" ne réfère peut-être pas au niveau $\mathrm{B} 2$, ensuite, le Cles revendique une démarche "holistique" intégrant en particulier la compréhension, la production et l'interaction orale (ce que ne faisait pas le Baccalauréat jusqu'à la session 2013).

On remarquera également que les descripteurs du Cecrl sont simplement transcrits, sans que soit explicitée la manière de lier les tâches aux niveaux de compétence. Mais c'est un autre sujet. Nous reproduisons ici le descripteur du deuxième degré correspondant au Cles 2, exigible en fin d'éducation supérieure :

Peut comprendre à l'écrit et à l'oral l'information explicite exprimée dans des messages complexes portant sur son domaine large de spécialité. Peut communiquer à l'écrit et à l'oral 
sur ce même domaine avec un degré de spontanéité et de fluidité qui rend aisés la compréhension et l'échange.

Rappelons en quoi consiste l'épreuve du Cles au niveau 2 :

Durée de l'épreuve: 3 heures (avec libre gestion du temps imparti pour les tâches de compréhension écrite et de production écrite) (+10 minutes d'interaction orale).

1/ Compréhension de l'oral

Tâches de compréhension globale et de repérage, puis traitement d'informations spécifiques à partir d'un ou plusieurs documents authentiques audio ou vidéo, d'environ 800 mots au total, traitant de sujets d'actualité liés aux grandes branches disciplinaires des enseignements supérieurs.

L'audition se fera en laboratoire de langue ou dans une salle équipée ad hoc.

2/ Compréhension de l'écrit

Tâches de compréhension globale et de repérage (notamment prise de notes guidée), puis traitement par hiérarchisation, agencement, etc., d'informations spécifiques à partir d'un ou plusieurs documents écrits authentiques totalisant environ 2000 mots, thématiquement liés aux documents d'écoute.

3/ Production écrite en situation

Rédaction d'un texte contextualisé d'environ 300 mots, avec mise en situation concrète et prise d'appui sur les documents d'écoute et de lecture.

4 / Interaction orale

8 à 10 minutes d'interaction orale à partir d'une mise en situation ayant un rapport avec les documents d'écoute et de lecture, en présence d'une examinateurobservateur-notateur.

Possibilité de concevoir cette épreuve sous forme d'interaction entre deux ou trois candidats (B.0. $\mathrm{n}^{\circ} 20 \mathrm{du} 17$ mai 2007)

\section{Forme}

21 Le Cles 2 emprunte la forme actionnelle du scénario, de la confrontation à un problème à la résolution duquel concourent les différentes activités de lecture, d'écoute, de prise de parole individuelle ou en interaction et d'écriture. Les éléments que nous avons soulignés en gras témoignent de la volonté des concepteurs du Cles de lier les parties de l'évaluation grâce à une thématique filée tout au long de l'épreuve par les différents supports de compréhension et de production. Est suggérée ici une forme d'homologie de l'évaluation et de l'apprentissage. Si l'évaluation a tendance à formater l'apprentissage en amont (washback effect), alors l'intérêt du Cles est évident. Il favorise l'implantation de méthodes d'enseignement fondées sur l'approche actionnelle ou approche par la tâche. On peut citer ici les différentes typologies de tâches non différenciables entre apprentissage et évaluation. L'un de ces typologies recensées par R. Ellis est la classification cognitive de Prabhu. Prabhu (1987) distingue trois types de tâches fondées sur la capacité cognitive impliquée : des activités proposant un déficit d'information, un déficit de raisonnement ou un déficit d'opinion. On comprend alors comment le Cles peut s'emparer de ce genre de typologie lorsque deux étudiants sont évalués en interaction (Prabhu, 1987 : 46, cité par Ellis, 2003 : 213) au niveau 2 et qu'il leur est demandé, par exemple, d'envisager de multiples points de vue sur une situation en fonction d'un rôle donné et d'interagir pour parvenir à un consensus final.

Ellis fait référence à un autre type de classification qui peut présider à la conception d'un projet pédagogique ou d'un scénario évaluatif : celle, psycholinguistique, de Pica, Kanagy, et Falodun, fondée sur les catégories interactionnelles :

- "La relation entre les interactants" (i.e. qui détient l'information, qui la recherche, etc.); 
- "les exigences de l'interaction" (i.e. l'information doit-elle être recherchée et fournie obligatoirement par les interactants?);

- "L’orientation du but" (i.e. la négociation de sens en vue du consensus ou du désaccord ?) ;

- "les options de résultat" (i.e. une seule issue possible ou plusieurs issues) (Pica, Kanagy et Falodun in Ellis, $2003: 215)$.

24 Ainsi, les épreuves de CLES 2 requièrent souvent que les partenaires de l'interaction parviennent à un consensus à partir d'un jeu de rôle "imposé" sur un sujet de controverse.

Ellis lui-même propose un cadre général en quatre points pour l'élaboration d'un cours s'appuyant sur l'approche par les tâches :

- "L’input", soit la nature des données fournies pour l'accomplissement de la tâche. Dans le cas du CLES, une thématique générale est proposée à laquelle tous les supports écrits et oraux renvoient. Il s'agit d'un input unifié ;

- "Les conditions", soit la manière dont l'information est présentée aux apprenants et la façon dont elle est utilisée. Les épreuves de CLES proposent des supports multimodaux;

- "Les processus sollicités", soit la nature des opérations cognitives en jeu et le type de discours requis par la tâche (informatif, explicatif, narratif, argumentatif). Le fait que le CLES soit une épreuve composite favorise la multiplicité des sollicitations tant au plan des opérations cognitives que des types de discours;

- "Les résultats", soit la nature du produit fini résultant de l'accomplissement de la tâche (Ellis, 2003 : 217).

On devine sans peine le fort potentiel d'homologie entre les scénarios d'apprentissage et le Cles.

\section{Une démarche holistique?}

27 Le Cles certifie-t-il vraiment "autrement" (selon la problématique initiale) ? Pour autant, s'agit-il véritablement d'une évaluation holistique, différente de celles pratiquées dans l'approche précédente?

Même si son utilisation confère une cohérence thématique, voire problématique, aux diverses tâches proposées, en dernier ressort, les différentes composantes de la compétence à communiquer langagièrement demeurent évaluées séparément. Ainsi, un étudiant qui valide le niveau B2 en compréhension de l'oral, en compréhension de l'écrit, en expression écrite, mais seulement le niveau B1 en interaction orale n'obtiendra pas le ClesS 2. D'un côté, ceci confirme que le CLES envisage le niveau de compétence de manière totalisante (l'échec d'une partie entraîne l'échec de l'ensemble), de l'autre, l'absence de compensation atteste que ce type de certificat n'adopte pas une démarche réellement holistique (la réussite de l'ensemble ne nécessite pas la réussite de chaque partie).

Pour résumer, le Cles appartient à une quatrième génération de test qui favorise un type d'apprentissage, proche de la vie réelle, sollicitant des compétences langagières intégrées à des processus cognitifs et discursifs repérables et susceptibles d'être développés en amont. Même si les compétences restent évaluées séparément comme dans le cas de la précédente approche des four skills, le gain est notable en termes de cohérence.

Pourquoi son existence est-elle remise en cause (par le décret de mai 2012 proposant de multiples alternatives à ce certificat et assorti d'une longue liste de cas de dispenses) ? On 
peut penser que la raison majeure est précisément cette homologie avec l'apprentissage : non seulement l'évaluation par le Cles requiert un grand nombre d'examinateurs et un nombre d'heures conséquents, mais encore, il induit des modes d'apprentissage, de complexité similaire, peu implantés dans les universités.

Un autre test, difficilement classable, pourrait venir s'interposer comme une alternative viable, le C-Test.

\section{Le C-Test}

\section{Définition}

Le C-Test est un test simple, fiable, valide, faisable et très économique (du fait de sa nature et de ses conditions de passation et de correction). C'est une forme améliorée du test de closure. Il s'agit de fournir un texte écrit dans lequel on efface la deuxième moitié du mot tous les 2 mots, tout en laissant intacte la première et la dernière phrase du texte. Chaque mot tronqué vaut pour un item d'évaluation. Un C-Test est généralement constitué de 4 à 6 textes et chaque texte contient 20 à 25 items, ce qui fait en tout environ 80 à 100 items par C-Test (Grotjahn, 2006 ; Reichert, 2011 : 40). Le candidat doit s'efforcer de reconstituer le texte au mieux. A titre indicatif, un locuteur natif réalise une performance de $90 \%$ en moyenne.

\section{Histoire}

Son origine remonte aux années 80 avec des expérimentations sur la redondance réduite ( reduced redundancy) menées par Klein-Braley $(1985,1997)$ et Digott (2004), cités par Reichert (2011: 45). Différents types de test furent mis au point, certains utilisant des sifflements (noise test) ou des dictées incomplètes. On trouve aussi des tests de closure à choix multiples où le testeur a enlevé certains mots qui doivent être replacés par le testé. Ce type de test en vogue pendant deux décennies fut abandonné pour des raisons liées à la validité et à la fiabilité.

\section{Renouveau}

Reichert souligne les améliorations notables apportées par le C-Test en termes de validité et de fiabilité précisément. Par exemple, le C-Test fournit beaucoup plus d'items sur un texte plus court, par rapport à un test de closure classique, ce qui rend le test plus fiable. De plus, le C-test est plus objectif, car il y a généralement très peu d'options autres que la solution originale, le scoring se faisant ainsi de manière plus neutre. Le C-Test continue à faire des émules dans de nombreuses langues. Applicable à tous types de publics pour tester la L1 aussi bien que la L2, il présente donc beaucoup de qualités. Autre intérêt, le CTest est le seul type de test que l'on ne peut préparer en amont, et par conséquent, qui ne peut pas formater l'apprentissage. En un sens, c'est un test "autre", une sorte d'alien dans le domaine, très différent du Cles, presque son antonyme.

Enfin, et c'est peut-être le paradoxe pour un test aussi peu "actionnel" dans sa forme même, il atteste, dans une certaine mesure, d'une compétence linguistique unifiée. Dans sa thèse, Reichert explique en effet que l'on s'est aperçu que les items avaient une forte corrélation entre eux, et donc que la compétence linguistique pouvait être unitaire 
conformément à la théorie développée par Oller (Reichert, 2011). Mais surtout, Reichert a montré le haut degré de corrélation entre les résultats au C-Test et ceux obtenus par les mêmes étudiants au Test de Connaissance du Français (TCF), de type Test of English as a Foreign Language (Toefl) amélioré comprenant l'évaluation en compréhension écrite et orale, en expression écrite et orale, et en compétence linguistique. Ce qui revient à dire qu'un test très facile à créer et très peu coûteux à faire passer fournit une information aussi fiable sur la compétence langagière (non sur la compétence communicative) d'un individu que d'autres tests beaucoup plus lourds. Certes, le C-Test ne fournit pas de renseignements précis sur chacune des compétences, ni sur les connaissances, et ne peut donc remplacer d'autres tests dont tel est l'objet, mais il peut tout à fait valider un niveau général et servir d'intermédiaire pour corréler d'autres tests entre eux. Ces premiers résultats obtenus par Reichert devraient susciter des travaux de consolidation dans le domaine.

\section{Conclusion}

De Ketele ajoute une dimension importante au cœur de notre problématique, à savoir, la fonction sociale de l'évaluation. Selon lui, évaluer c'est confronter un ensemble d'informations à un ensemble de critères (référentiel) en vue de prendre une décision (De Ketele, 1980). De quelle décision parle-t-on ici ? S'agit-il d'une décision personnelle de l'apprenant, de celle du professeur ou du formateur évaluateur, d'un jury d'examen, d'un employeur? Par définition, une évaluation distanciée de type certification se situe hors de la sphère familière de la classe, et implique une prise de décision qui échappe au sujet évalué. Plus le besoin d'un regard distancié est affirmé, plus le sujet semble devoir être mis à distance de la prise de décision, avec des effets potentiellement nuisibles pour lui.

Gardner soulève aussi la question et définit deux destinataires des informations obtenues par l'évaluation: "Evaluer, c'est recueillir des données sur les compétences et les potentiels des individus dans le double objectif de leur en faire retour utilement et de procurer des informations indispensables à la communauté environnante" (Gardner, 1996 : 114).

Si l'on veut vraiment privilégier l'aspect individuel et social de l'évaluation, il conviendrait d'afficher clairement une éthique. Dans ces conditions, les informations recueillies sur chaque apprenant devraient lui être retournées sur le mode de son choix (appréciation, note, couleur, diplôme, etc.) : elles lui appartiennent de droit en tant que sujet. La société qui souhaite obtenir des résultats plus précis sur les compétences langagières pourrait se satisfaire de l'information pourvue par le référentiel correspondant à la session d'apprentissage, ou d'une estimation empirique du niveau de cette session. Une alternative consisterait à offrir des certifications, nécessitant un appareil plus lourd comme le Cles ou plus léger comme le C-Test. Au final, Cles et C-Test aussi étrangers par rapport aux autres types de tests que l'un à l'autre semblent non seulement compatibles (le C-test apparait comme une possibilité dans le descriptif du Cles 1 et complémentaires mais pourraient s'avérer utiles, de manière égale, au sujet plurilingue et à la société. 


\section{BIBLIOGRAPHIE}

Alderson, J. C. (2002). "Testing proficiency and achievement: principles and practice". In Coleman, J. A., Grotjahn, R. and Raatz, U. (eds) University language testing and the C-Test. Bochum: AKS Verlag, pp. 15-30.

Alderson, J. C., Figueras, N., Kuiper H., Nold G., Takala S., Tardieu, C., (2006). “Analyzing Tests of Reading and Listening in Relation to the Common European Framework of Reference: The experience of the Dutch Grid Construct Project". In Language Assessment Quarterly, vol. $3 \mathrm{n}^{\circ} 1$, pp. 3-30.

A.L.T.E (2011). Manual for Language Test Development and Examining. Language Policy Division, Council of Europe. Disponible en ligne : http://www.coe.int/t/dg4/linguistic/ ManualtLangageTest-Alte2011_EN.pdf Anderson, N. (1996). A Functional Theory of Cognition. Psychology Press.

Bachman, L.F. (1990). Fundamental Considerations in Language Testing. Oxford, UK: Oxford University Press.

Baccalauréat:

- B.O.E.N. $n^{\circ} 27$ du 7 juin 1983

- B.O.E.N. $n^{\circ} 23$ du 7 juin 2001

Bachman, L.F. (2007). "What is the construct? The dialectic of abilities and contexts in defining constructs in language assessment". In J. Fox, M. Wesche, D. Bayliss, L. Cheng, C.E. Turner \& C.Doe (Eds.), Language testing reconsidered. Ottawa, CA: University of Ottawa Press. pp. 41-72.

CLES:

B.O.E.N. $n^{\circ} 25$ du 29 juin 2000 (http://www.education.gouv.fr/bo/2000/25/sup.htm).

Décret n 2012-999 : http://www.education.gouv.fr/cid56637/certificat-informatique-internetexige-compter-session-2012.html

B.O.E.N. $n^{\circ} 20$ du 17 mai 2007

(http://www.education.gouv.fr/bo/2007/20/MENS0700723A.htm)

Bolton, S. (1991). Evaluation de la compétence de communication en langue étrangère. Coll. "Langues et Apprentissage des Langues". Paris: Credif-Hatier/Didier.

Canale, M. \& Swain, M. (1980) "Theoretical bases of communicative approaches to second language teaching and testing”. Applied Linguistics , 1, 1-47.

De Ketele, J.-M. (1980). Observer pour éduquer. Préf. de M. Postic. Berne : Peter Lang.

Ellis, R. (2003). Task-based Language Learning and Teaching. Oxford: Oxford University Press.

Grotjahn, R., Klein-Braley, C. \& Raatz, U. (2002). “C-Tests: an overview”. In J.A. Coleman, R. Grotjahn \& U. Raatz (Eds.), University Language Testing and the C-Test. Bochum, Germany : ASKVerlag. pp. 93-114.

Horner, D. (2010). Le CECRL et l'évaluation de l'oral en anglais. Paris : Belin, Coll. Guides de l'enseignement. 
Laugier, H. \& D. Weinberg (1938). Recherches sur la solidarité et l'indépendance des aptitudes intellectuelles d'après les notes des examens écrits au baccalauréat. Paris.

McNamara, T. (2000). Measuring second language performance. Oxford: Oxford University Press.

Oller, J.W. (1976). "Evidence for a general language proficiency factor: an expectancy grammar". Die Neuren Sprachen, 75, pp. 165-174.

Pica, R., Kanagy, R. \& Falodun, J. (1993). “Choosing and using communication tasks for second language research and instruction". In S. Gass \& G. Crookes (eds.) Task-based Learning in a Second Language. Clevedon : Multilingual Matters.

Piéron, H. (1963). Examens et Docimologie. Paris : P.U.F.

Prabhu, N.S. (1987). Second Language Pedagogy. Oxford: Oxford University Press.

Reichert, M. (2011). The validity of the C-Test revisited: findings from a multilingual environment. Thèse de doctorat non publiée, Université du Luxembourg, Luxembourg.

Springer, C. (2000). "Les multiples facettes du concept de "compétence" : que peut-on en retenir pour l'enseignement et l'apprentissage des langues ?". In Cognition, langue et culture, éléments de théorisation didactique. Actes de la journée d'étude du 17 mars 2000. GEPED, Université Paris7 Denis Diderot.

\section{RÉSUMÉS}

Au sein de la thématique "Apprendre les langues autrement", cet article se focalisera sur la question de la certification en langues au moment où les modes d'évaluation ont tendance à évoluer et où des certifications européennes et internationales viennent supplanter les diplômes nationaux. On s'intéressera en particulier à deux certificats que tout semble opposer: Le Certificat de Compétences en Langues de l'Enseignement Supérieur (Cles) et le C-Test (forme spécifique de l'exercice de closure).

Within the theme "Learning Languages differently", this paper focuses on the issue of language certification at the very moment when the means of assessment tend to evolve and different European and international certificates are superseding national diplomas. We will deal especially with two certificates which are seemingly incompatible : the Certificat de Compétence en Langues de l'Enseignement Supérieur (Cles) (The Language Certificate of Higher Education) and the C-Test (a specific type of cloze test).

\section{INDEX}

Keywords : language certification, Cles, C-Test, testing

Mots-clés : certification en langue, Cles, C-Test, testing

Thèmes : Varia

\section{AUTEUR}

\section{CLAIRE TARDIEU}

Claire Tardieu est professeur de didactique de l'anglais à l'université Sorbonne Nouvelle Paris 3. Elle participe à l'évaluation des élèves en fin de troisième à la Division de 
l'Évaluation de la Prospective et de la Performance du Ministère de l'Éducation Nationale et a représenté la France dans trois projets européens dans le domaine de l'évaluation et de la certification en langues : Dutch Cefr grid, Ebafls et CEF-Estim.

Courriel : claire.tardieu@univ-paris3.fr 